\title{
FIELD EQUATIONS AND EQUATIONS OF MOTION IN POST-NEWTONIAN APPROXIMATION OF THE PROJECTIVE UNIFIED FIELD THEORY*
}

\author{
ALEXANDER GORBATSIEVICH \\ Department of Physics of Belorussian State University, Nesavisimosti av., \\ Minsk, 220030, Belarus \\ Gorbatsievich@bsu.by \\ ERNST SCHMUTZER \\ Friedrich Schiller University of Jena \\ D-07743 Jena, Germany \\ eschmutzer@t-online.de
}

\begin{abstract}
The equations of motion of $N$ gravitationally bound bodies are derived from the field equations of Projective Unified Field Theory. The Newtonian and the post-Newtonian approximations of the field equations and of the equations of motion of this system of bodies are studied in detail. In analyzing some experimental data we performed some numeric estimates of the ratio of the inertial mass to the scalaric mass of matter.
\end{abstract}

\section{Introduction}

Recently considerable interest raised by experiments aiming at the verification of the relativistic theories of gravitation (in detail see 1 and the literature quoted there). The high precision experiments on checking the validity of the equivalence principle, perihelion shift of Mercury, deflection of light and other effects give some answer to the question of preference among further gravitational theories proposed, where the distinction between them is mainly based on the post-Newtonian or even on the post-post-Newtonian level.

Among the alternative theories of gravitation here our special interest is directed to the 5-dimensional Projective Unified Field Theory of Schmutzer (short:

*Dedicated to Prof. Dr. Nikolay Mitskievich on the occasion of his $80^{\text {th }}$ birthday. 
PUFT). For details of the axiomatics as well as the cosmological and astrophysical application of PUFT see [2 4] and [8. Within the framework of this theory recently interesting cosmological models were investigated [4, 9, where in particular an unexpected solution of the problem of dark matter was suggested. For some observable cosmological effects see also [10. Obviously within the framework of PUFT the systematic study and subsequent empirical testing of various astrophysical effects is necessary, particularly effects in the solar system. First steps in this direction were made in the papers of E. Schmutzer quoted above. However the wishes of astrophysicists, particularly of those being active researchers in celestial mechanics, ask for a systematic treatment of this problem by building up the post-Newtonian approximation of PUFT.

Schmutzer's PUFT has been developed in three stages: The version I of PUFT (1958) led to a possible violation of the equivalence principle which nowadays has already been tested experimentally to a relative precision of $10^{-12} \div 10^{-14}$ [1]. Two decades later this situation was one of the reasons for him to elaborate the version II of PUFT (GR9-conference in Jena 1980), where a projection formalism with a kind of "conformal projection factor" was used. Emphasizing his 5-dimensional concept of describing real physics, intended since the beginning of his research in this field (see [11]), he already then introduced the concept of scalarism (scalarity) as a hypothetical new phenomenon of Nature: PUFT unifying gravitation, electromagnetism and scalarism.

Detailed information on this historical development of PUFT and present state of PUFT can also be found in the monographs [3,4] and [3, 7], respectively.

In the following part of this paper we develop the post-Newtonian approximation of PUFT (field equations, equation of motion of a test body and corresponding astrophysical applications). Our results allow to compare the predictions of PUFT and the Einstein theory with the experimental data.

\section{Four-dimensional field equations and mechan- ical equations of motion (continuum and point- like test body)}

\subsection{Field equations in the space-time}

The 4-dimensional field equations of PUFT were received by projecting the 5 -dimensional field equations of the projective space onto 4-dimensional spacetime. In the following we present the 4-dimensional field equations of PUFT in the Gauss system of units (Latin indices run from 1 to 4, Greek indices from 1 to 3 ; the signature of the metric is $(+,+,+,-)$; comma and semicolon denote partial and covariant derivatives, respectively) [2 4].

\subsubsection{Generalized gravitational field equation}

$$
R^{m n}-\frac{1}{2} g^{m n} R-\Lambda_{\mathrm{S}} \mathrm{e}^{-2 \sigma} g^{m n}=\varkappa_{0}\left(E^{m n}+S^{m n}+\Theta^{m n}\right) .
$$


Here

$$
R_{m n}=R_{m n i}^{i} \quad \text { and } \quad R=R_{m}^{m}
$$

are the usual 4-dimensional curvature quantities. Further one should realize the cosmological term on the left-hand side of the field equation, being the analog to that of the Einstein theory (General Relativity), but here the scalaric field $\sigma$ is involved. Further $\Lambda_{\mathrm{S}}$ is the scalaric-cosmological constant in PUFT with physical dimension of reciprocal square of length.

In the basic field equations of Schmutzer's 5-dimensional PUFT a cosmological function is present, instead of the cosmological constant of Einstein's 4-dimensional field theory. Here we should mention that this situation is similar to the ansatzes of the quintessence in the cosmological models being intensively investigated at present time.

Let us in this context point to the relation

$$
\varkappa_{0}=\frac{8 \pi \gamma_{\mathrm{N}}}{c^{4}}
$$

used by Schmutzer under following aspects: Subject to eventual later theories with this Einstein gravitational constant $\varkappa_{0}$, as well the Newtonian gravitational constant $\gamma_{\mathrm{N}}$ and the vacuum velocity of light $c$ are true constants of Nature. With respect to the numerical values, of course, one has to be careful, since according to the precise measurements of the last decade it seems that the measured "Newton gravitational parameter" $G$ is a time-dependent quantity. Our subsequent treatment of the post-Newtonian approximation shows a conceivable relationship between these two quantities $\gamma_{\mathrm{N}}$ and $G$. In the present time the numerical values of both are closely neighboring.

The energy-momentum tensor of the non-geometrized matter (substrate) is denoted by $\Theta^{m n}$. Further the following notations are used:
a) $E^{m n}=\frac{1}{4 \pi}\left(B^{m k} H_{k}^{n}+\frac{1}{4} g^{m n} B_{k l} H^{k l}\right)$
(electromagnetic energy-
b) $S^{m n}=\frac{2}{\varkappa_{0}}\left(\sigma^{, m} \sigma^{, n}-\frac{1}{2} g^{m n} \sigma_{, k} \sigma^{, k}\right)$
(scalaric energy-momentum tensor).

The electromagnetic field strength tensor $B_{i j}$ and the electromagnetic induction tensor $H_{i j}$ will be explained in context with the electromagnetic field equations.

As Schmutzer earlier pointed out, in the electromagnetic situation it is physically suggestive (without changing the physical content) to absorb the occurring factor on the right-hand side of the equation (4b) by introducing instead of the dimensionless scalaric field function $\sigma$ the modified scalaric field function (with a corresponding physical dimension) $\hat{\sigma}=\sqrt{\frac{2}{\varkappa_{0}}} \sigma$. But with respect to the approximation procedure we will keep to the more convenient $\sigma$. 
Specialization to a perfect fluid gives in concretization of the energy-momentum tensor $\Theta^{m n}$ the result

$$
\Theta^{m n}=-\left(\mu+\frac{p}{c^{2}}\right) u^{m} u^{n}-p g^{m n} \quad \begin{aligned}
& \left(u^{m} \text { four-velocity, } \mu \text { mass density, } p\right. \text { (5) } \\
& \text { pressure) }
\end{aligned}
$$

(The minus sign is connected with the definition (2) of Ricci tensor.)

\subsubsection{Generalized electromagnetic field equations}

These basic equations read as follows:
a) $H_{; n}^{m n}=\frac{4 \pi}{c} j^{m} \quad$ (inhomogeneous system),
b) $B_{<i j, k>}=0 \quad$ (cyclic system),
c) $H^{m n}=\varepsilon B^{m n} \quad$ with
d) $\varepsilon=\mathrm{e}^{2 \sigma} \quad$ (vacuum dielectricity/polarisation).

The quantity $j^{m}$ means the electric current density, e.g. in the convective case: $j^{m}=\varrho u^{m}$, where $\varrho$ is the charge density.

\subsubsection{Scalaric field equation}

This equation has the form:

$$
\sigma^{, k}{ }_{; k}-\Lambda_{\mathrm{S}} \mathrm{e}^{-2 \sigma}=-\frac{\varkappa_{0}}{2}\left(\frac{1}{8 \pi} B_{i j} H^{i j}+\vartheta\right) .
$$

In this equation the scalaric substrate energy density $\vartheta$ (short: scalerg density) occurs, which is a basically new quantity within the framework of the traditional 4-dimensional physics (see [3,4]). Later we shall try to interpret it.

\subsubsection{Equation of motion of the electrofluid and electric continuity equation}

Analogously to the procedure in the Einstein theory, by covariant differentiation of the above field equations (11) and (6a) we receive the following balance equations:

$$
\text { a) } \Theta_{; n}^{m n}=-\frac{1}{c} B_{k}^{m} j^{k}+\vartheta \sigma^{, m}, \quad \text { b) } j_{; m}^{m}=0 .
$$

For a perfect electrofluid by means of (5) the corresponding equation of motion results:

$$
\begin{array}{r}
\left(\mu+\frac{p}{c^{2}}\right) u_{; k}^{m} u^{k}=\frac{\varrho}{c} B_{k}^{m} u^{k}-\left(p^{, m}+\frac{1}{c^{2}} \frac{\mathrm{d} p}{\mathrm{~d} \tau} u^{m}\right) \\
-\vartheta\left(\sigma^{, m}+\frac{1}{c^{2}} \frac{\mathrm{d} \sigma}{\mathrm{d} \tau} u^{m}\right) .
\end{array}
$$




\subsection{The equations of motion of a point-like test body}

As we realized, the equation of motion in PUFT (as in the Einstein theory) can be derived from the field equations. Of course, its explicit form requires concrete assumption for the energy-momentum tensor $\Theta^{m n}$, electric four-current density $j^{k}$ and the scalerg density $\vartheta$.

As it is well-known, in the Einstein theory for incoherent matter $\Theta^{m n}=$ $-\mu u^{m} u^{n}$ and $j^{m}=\rho u^{m}$ ( $\mu$ mass density, $\rho$ electric charge density). This means for the case of a point-like test body (material point) with the inertial mass $M$ and charge $Q$ that the following formulas are valid:

$$
\begin{aligned}
& \Theta^{m n}=-c \int \frac{M \delta^{4}(x-\xi(\tau)) u^{m} u^{n}}{\sqrt{g(\xi(\tau))}} \mathrm{d} \tau, \\
& j^{m}=Q c \int \frac{\delta^{4}(x-\xi(\tau)) u^{m}}{\sqrt{g(\xi(\tau))}} \mathrm{d} \tau,
\end{aligned}
$$

where the integrals in the equations (10) and (11) must be taken along the world line (determined by $\left.x^{i}=\xi^{i}(\tau)\right)$ of the test body; $u^{m}=\frac{\mathrm{d} \xi^{m}}{\mathrm{~d} \tau}$ is the 4-velocity of the test body and $g=-\operatorname{det}\left(g_{i j}\right)$. By substituting (10) and (11) into equation (8) we obtain the condition

$$
\vartheta \sigma^{, i}=-c \int \frac{\delta^{4}(x-\xi(\tau))}{\sqrt{g(\xi(\tau))}}\left[\frac{\mathrm{D}\left(M u^{i}\right)}{\mathrm{D} \tau}-\frac{e}{c} B_{k}^{i} u^{k}\right] \mathrm{d} \tau,
$$

from wich the following structure of $\vartheta$ follows directly:

$$
\vartheta=c^{3} \int \frac{\mathcal{M} \delta^{4}(x-\xi(\tau))}{\sqrt{g(\xi(\tau))}} \mathrm{d} \tau .
$$

The multiplier $c^{3}$ in the expression (13) guarantees that the new introduced quantity $\mathcal{M}$, which is called "scalaric mass" (scalmass), has the same physical dimension as the inertial mass $M$. Therefore the quantity $D=\mathcal{M} c^{2}$ is named "scalaric substrate energy" (short: scalerg [2]).

By substituting (10), (11) and (13) into equations (8) we obtain

$$
\frac{\mathrm{D}\left(M u^{i}\right)}{\mathrm{D} \tau}=\frac{Q}{c} B_{k}^{i} u^{k}-c^{2} \mathcal{M} \sigma^{, i} .
$$

Multiplying (14) by $u_{i}$ and keeping in mind that $u^{i} u_{i}=-c^{2}$, instead of (14) we find the following equations which describe the motion of a point-like test body in a gravitational, electromagnetic and scalaric field:

$$
\begin{gathered}
M \frac{\mathrm{D} u^{i}}{\mathrm{D} \tau}=\frac{e}{c} B_{k}^{i} u^{k}-c^{2} \mathcal{M} P^{i}{ }_{j} \sigma^{, j}, \\
\frac{\mathrm{d} M}{\mathrm{~d} \tau}=\mathcal{M} \sigma^{, k} u_{k}=\mathcal{M} \frac{\mathrm{d} \sigma}{\mathrm{d} \tau},
\end{gathered}
$$


where $P_{j}^{i}$ is the projection tensor,

$$
P_{k}^{i}=g_{k}^{i}+\frac{1}{c^{2}} u^{i} u_{k}, \quad P_{k}^{i} u^{k}=0 .
$$

We mention that the equations (15) and (16) coincide with the corresponding ones performing a transition from the equation of motion of a perfect fluid [2]. From the equation (16) follows that there exists a dependence of the inertial mass (with particular features) of the test body in PUFT. Further one realizes that in the case of a vanishing scalaric field PUFT goes over into the Einstein theory [2] in which $M=M_{0}=$ const. Therefore as a consequence of (16) it is quite natural to suppose that the variability of the inertial mass is exclusively caused by the scalaric field, i.e. $M=M(\sigma)$. Hence follows that the scalaric mass is determined, in correspondence with equation (16), by $\mathcal{M}=\frac{\mathrm{d} M(\sigma)}{\mathrm{d} \sigma}$. In the case of an electrically neutral particle $(Q=0)$ or in the absence of an external electromagnetic field $\left(B_{m n}=0\right)$ equation (15) reads:

$$
\frac{\mathrm{D} u^{i}}{\mathrm{D} \tau}=-c^{2} \eta(\sigma)\left(\sigma^{, i}+\frac{1}{c^{2}} \sigma^{, k} u_{k} u^{i}\right),
$$

where $\eta(\sigma)=\frac{\mathcal{M}(\sigma)}{M(\sigma)}$. The equation (18) according to the concept of Schmutzer [2] contains an arbitrary function $\eta(\sigma)$.

Let us note that we can give another interpretation of the both masses $M$ and $\mathcal{M}$. In particular we write

$$
M(\sigma)=M_{0} f(\sigma) \quad \text { and } \quad \mathcal{M}=M_{0} f^{\prime}(\sigma) \quad\left(M_{0}=\text { const }\right),
$$

where $f(\sigma)$ as yet is an arbitrary function of the $\sigma$-field. Further we will call the constant $M_{0}$ inertial mass of the point-like body. Thus as well as in the general relativity the inertial mass remains a constant, but the equations of motion change:

$$
M_{0} \frac{\mathrm{D} u^{i}}{\mathrm{D} \tau}=-c^{2} \mathcal{M} P_{j}^{i} \sigma^{, j} \quad\left(\mathcal{M}=M_{0} \frac{f^{\prime}(\sigma)}{f(\sigma)}\right) .
$$

Obviously, both approaches are mathematically equivalent. But the second approach (see (20) ) is preferable from the physical point of view.

Let's note that equations of motion (20) can be rewritten in the form of Lagrange equations

$$
\frac{\mathrm{d}}{\mathrm{d} \tau}\left(\frac{\partial \mathcal{L}}{\partial \dot{x}^{i}}\right)-\frac{\partial \mathcal{L}}{\partial x^{i}}=0, \quad \dot{x}^{i}=\frac{\mathrm{d} x^{i}}{\mathrm{~d} \tau}
$$

with

$$
\mathcal{L}=-M_{0} c f(\sigma) \sqrt{-g_{i j} \dot{x}^{i} \dot{x}^{j}}+\frac{Q}{c} A_{i} \dot{x}^{i},
$$


where $A_{i}$ is the electromagnetic 4-potential. For the electrically neutral bodies $(Q=0)$ the equations of motion are equivalent to the equations of time-like geodesics in the pseudo-Riemannian space with the conformally transformed metric

$$
g_{i j} \quad \rightarrow \quad \tilde{g}_{i j}, \quad \text { where } \tilde{g}_{i j}=f^{2}(\sigma) g_{i j} .
$$

Let as mention that from (20) one immediately learns that the weak equivalence principle is fulfilled exactly if $f(\sigma)$ is a universal function for all kind of matter. Obviously, given relation for extended bodies can be fulfilled only approximately as $M$ and $\mathcal{M}$ have the different physical nature.

The post-Newtonian approximation of PUFT for the case

$$
M(\sigma)=M_{0} e^{\eta_{0} \sigma}, \quad \mathcal{M}(\sigma)=\left(\eta_{0} M_{0}\right) \mathrm{e}^{\eta_{0} \sigma}, \quad\left(\eta_{0}=\text { const }\right)
$$

was constructed in the paper [12]. In this context we note that just the dependence between the inertial and scalaric masses in the form

$$
M(\sigma)=\mathcal{M} \sigma, \quad \mathcal{M}(\sigma)=\text { const },
$$

and some other dependencies were studied and elaborated in detail by Schmutzer for various cosmological models. The results of this cosmological investigations are resumed in the monographs [4, 9 .

\section{Post-Newtonian Approximation of the PUFT}

\subsection{Introduction}

Let us consider a system of slowly moving bodies bounded by gravitational interaction (e.g. planetary system). In order to describe the gravitational field of such a system at large distances from its center, in the Einstein theory one can use the so-called post-Newtonian approximation. Here we will show that the field equations of PUFT also allows an analogous approximation. Similar to the procedure in the Einstein theory it is convenient to take the ratio $\beta=$ $v / c$ as a small expansion parameter of the exact field equations, where $v$ is the characteristic velocity of the motion of the bodies, which is related to the gravitational potential $\phi$ as follows:

$$
\frac{v^{2}}{c^{2}} \sim \frac{\phi}{c^{2}} \sim \beta^{2}
$$

(in the planetary system $\beta \sim 10^{-4}$ to $10^{-3}$ ).

For the further investigation we restrict our considerations to the following assumptions:

1. The electromagnetic field is equal to zero: $B^{i j}=0$. 
2. According to the suppositions (19) and (20) we assume that

$$
\frac{f^{\prime}(\sigma)}{f(\sigma)} \ll 1
$$

for all bodies.

3. The energy-momentum tensor of the perfect fluid has the standard form (5)

4. For the system considered the equations (7) are joint with the assumption that the scalaric field may be introduced as a superposition of two fields

$$
\sigma=s+\bar{\sigma} \quad(|s| \ll|\bar{\sigma}|),
$$

whose first one $(s)$ has sources inside the considered system of bodies, and the second one $(\bar{\sigma})$ refers to the outside of it. In particular, $\bar{\sigma}$ may have a cosmological origin. Thus by consideration of the motion of bodies inside the planetary system we may regard the field $\bar{\sigma}$ as a quasi constant field: $(\bar{\sigma})_{, k} \approx 0$.

5. We suppose that there exists a coordinate system, in which in zeroth order approximation the metric tensor equals the Minkowski tensor $\eta_{i j} \equiv$ $\operatorname{diag}(1,1,1,-1)$. Then the following power series approximation is possible:

$$
\begin{aligned}
& g_{\alpha \beta}=\delta_{\alpha \beta}+\stackrel{2}{g}_{\alpha \beta}+O\left(\beta^{4}\right), \\
& g_{\alpha 4}=\stackrel{3}{g}_{\alpha 4}+O\left(\beta^{5}\right), \\
& g_{44}=-1+\stackrel{2}{g}_{44}+\stackrel{4}{g}_{44}+O\left(\beta^{6}\right) ;
\end{aligned}
$$

and for the stress tensor $T^{m n} \equiv \Theta^{m n}+S^{m n}+E^{m n}$ :

$$
\begin{aligned}
& T^{\alpha \beta}=\stackrel{0}{T^{\alpha \beta}}+\stackrel{2}{T}^{\alpha \beta}+O\left(\beta^{4}\right), \\
& T^{\alpha 4}=\stackrel{1}{T^{\alpha 4}}+O\left(\beta^{5}\right), \\
& T^{44}=\stackrel{0}{T^{44}}+\stackrel{2}{T}^{44}+\stackrel{4}{T^{44}}+O\left(\beta^{6}\right) .
\end{aligned}
$$

\subsection{Newtonian-like approximation of PUFT}

In the case of a vanishing electromagnetic field the equations (1) and (7) in consideration of (28) lead to the Newtonian-like approximation:

$$
\text { a) } \triangle \phi=4 \pi \gamma_{\mathrm{N}} \mu, \quad \text { b) } \triangle s=-\frac{4 \pi \gamma_{\mathrm{N}}}{c^{2}} \mu^{(\sigma)} \text {, }
$$

where $\phi$ is the gravitational potential, which is connected with the metric:

$$
g_{44}=-1-\frac{2 \phi}{c^{2}}
$$


$\mu=-\frac{1}{c^{2}} \stackrel{0}{T}^{44}$ is the mass density, and $\mu^{(\sigma)}=\frac{1}{c^{2}} \vartheta$ is the so-called scalaric mass density (scalmass density). In the case of $N$ gravitationally bounded point-like bodies the quantities $\mu$ and $\mu^{(\sigma)}$ in the Newtonian-like approximation take the form ( $A$ running from 1 to $N$ )
a) $\mu=\sum_{A} M_{A} \delta^{(3)}\left(\boldsymbol{r}-\boldsymbol{r}_{A}\right)$
b) $\mu^{(\sigma)}=\sum_{A} \mathcal{M}_{A} \delta^{(3)}\left(\boldsymbol{r}-\boldsymbol{r}_{A}\right)$.

The solution of the equations (31) which vanishes at infinity can be written as

$$
\text { a) } \phi\left(\boldsymbol{r}_{K}\right)=-\gamma_{\mathrm{N}} \sum_{A \neq K} \frac{M_{A}}{r_{A K}}, \quad \text { b) } s\left(\boldsymbol{r}_{K}\right)=\gamma_{\mathrm{N}} \frac{1}{c^{2}} \sum_{A \neq K} \frac{\mathcal{M}_{A}}{r_{A K}},
$$

where the abbreviations

$$
r_{A K}=\left|\boldsymbol{r}_{A K}\right|, \quad \boldsymbol{r}_{A K}=\boldsymbol{r}_{A}-\boldsymbol{r}_{K}
$$

are used.

Taking into account the assumption (28) and the solutions (34), we obtain the following equations of motion for $N$ gravitationally bounded bodies in the Newtonian-like approximation of PUFT:

$$
\frac{\mathrm{d} \boldsymbol{v}_{K}}{\mathrm{~d} t}=-\gamma_{\mathrm{N}} \sum_{A \neq K} M_{A}\left(1-\frac{\mathcal{M}_{A}}{M_{A}} \frac{\mathcal{M}_{K}}{M_{K}}\right) \frac{\boldsymbol{r}_{K}-\boldsymbol{r}_{A}}{\left|\boldsymbol{r}_{K}-\boldsymbol{r}_{A}\right|^{3}} .
$$

Let us notice that up to now both the inertial mass $M$ and the scalaric mass $\mathcal{M}$ are in general completely independent. However for fulfilling the weak equivalence principle it is necessary that the ratio of both masses is for all bodies the same universal function of the scalaric field. As it was mentioned above, the equivalence principle has already been tested experimentally to a relative precision of $10^{-12} \div 10^{-14}$ [1]. Thus the function $f(\sigma)$ (see (19) ) at least in the Sun-system is in very good approximation an universal function: $\frac{\left|f_{K_{1}}-f_{K_{2}}\right|}{f_{K_{1}}}<10^{-12}$. Taking into account the assumptions (28) and (27) we can write for all bodies

$$
M_{K}(\sigma)=f(\sigma) M_{0 K}, \quad f(\sigma) \approx f(\bar{\sigma})\left(1+\frac{f^{\prime}(\bar{\sigma})}{f(\bar{\sigma})} s\right) .
$$

With the help of the last relation we can put the equation of motion into the Newtonian-like form

$$
\frac{\mathrm{d} \boldsymbol{v}_{K}}{\mathrm{~d} t}=-G_{\mathrm{S}} \sum_{A \neq K} M_{A} \frac{\boldsymbol{r}_{K}-\boldsymbol{r}_{A}}{\left|\boldsymbol{r}_{K}-\boldsymbol{r}_{A}\right|^{3}}=-G_{\mathrm{S}} \sum_{A \neq K} M_{0 A} f(\bar{\sigma}) \frac{\boldsymbol{r}_{K}-\boldsymbol{r}_{A}}{\left|\boldsymbol{r}_{K}-\boldsymbol{r}_{A}\right|^{3}},
$$

where we have introduced the "scalaric-gravitational parameter" $G_{\mathrm{S}}($ see $[3,4])$ :

$$
G_{\mathrm{S}} \equiv \gamma_{\mathrm{N}}\left(1-\delta^{2}\right) \approx 6.6726 \times 10^{-8} \frac{\mathrm{cm}^{3}}{\mathrm{~g} \mathrm{~s}^{2}},
$$


which numerically coincide at the present time with the conventional Newtonian gravitational constant. Here

$$
\delta=\frac{f^{\prime}(\bar{\sigma})}{f(\bar{\sigma})}=\frac{\mathcal{M}(\bar{\sigma})}{M(\bar{\sigma})} \simeq \text { const } \quad(\bar{\sigma}=\bar{\sigma}(t)), \quad \delta \ll 1 .
$$

Summarizing the last results, we can write the field equations (311) and the equation of motion of a test point-like body in the Newtonian-like approximation of PUFT in the familiar form:

$$
\text { a) } \frac{\mathrm{d} \boldsymbol{v}}{\mathrm{d} t}=-\nabla \Phi_{\mathrm{eff}}, \quad \text { b) } \triangle \Phi_{\mathrm{eff}}=4 \pi G_{\mathrm{S}} \mu,
$$

using the quantity

$$
\Phi_{\mathrm{eff}}=\phi+c^{2} \frac{f^{\prime}(\bar{\sigma})}{f(\bar{\sigma})} s=\phi+c^{2} \delta s
$$

as effective (empirical) Newtonian potential.

From these considerations we learn that the scalaric-gravitational parameter $G_{\mathrm{S}}$ is not a constant in strict sense, since it depends on the scalaric field $\bar{\sigma}$ which was introduced for the description of the effective cosmological influence. Therefore $\dot{G}_{\mathrm{S}} \equiv \frac{\mathrm{d} G_{\mathrm{S}}}{\mathrm{d} t} \neq 0$, but $\frac{\dot{G}_{\mathrm{S}}}{G_{\mathrm{S}}}$ is very small. Because of this extreme smallness up to now direct measurements of $\frac{\dot{G}_{\mathrm{S}}}{G_{\mathrm{S}}}$ in laboratories are without success. But nevertheless for the Earth-Moon system indirect estimates of $\frac{\dot{G_{S}}}{G_{\mathrm{S}}}$, received as result of evaluating the motion of the lunar orbit, give magnitudes till $10^{-14} \div 10^{-13} \mathrm{~s}^{-1}$, particularly E. V. Pitjeva: $10^{-14} \mathrm{~s}^{-1}$ [13]. However one should remember that these numerical results are received within the framework of Newtonian celestial mechanics, being corrected by admitting a timedepending gravitational constant. Of course, in the case of PUFT the evaluation of the same observed data can yield essentially differing results, being of high importance for the numerical proof of PUFT.

Let us in this context emphasize that the equation (31b) was obtained for the case of vanishing external electromagnetic fields from the basic equation (77), in which as source term for the scalaric field also the (electromagnetic) Larmor invariant $\Lambda_{\mathrm{L}}=B_{i j} H^{i j}$ occurs. In the previous calculations this term has been neglected. But for deeper understanding of the electromagnetic influence on scalarism the Larmor term has to be taken into account. For example, this term should not be ignored for a detailed treatment of the inner region of electrically neutral matter, where local electromagnetic fields of appreciable strength occur, inducing local scalaric fields. According to (7) these local scalaric fields $\sigma_{\text {loc }}$ may yield a considerable contribution to the global $\sigma$-field appearing in (31b).

Let us further mention that recently a review article on various 4-dimensional approaches of theories with time-dependent cosmological gravitational parameters appeared, comparing the ansatzes with corresponding measuring results (see [14]). 
Concluding this subsection, let us draw our attention to another interesting subject. As it is well known, the first measurement of the frequency red shift in a gravitational field (experiments of Pound and Rebka [15] and Pound and Snider [16]) shows that the free motion of bodies corresponds to geodesics up to very high precision. Looking at (18) one immediately recognizes that in the PUFT the free fall is not the geodesic motion. This fact means that PUFT is a non-metric gravitational theory in the sense of this definition. In the Newtonianlike approximation we can say that the red shift is determined by the metric, i.e. by the gravitational potential $\phi$ (see (32))

$$
\frac{\Delta \nu}{\nu}=-\frac{\Delta \phi}{c^{2}}=-\left(1-\delta^{2}\right)^{-1} \frac{\Delta \Phi_{\mathrm{eff}}}{c^{2}} .
$$

In contrast to this result the gravitational force depends on the effective Newtonian potential $\Phi_{\text {eff }}$. In the conventional notation the red shift formula has the shape

$$
\frac{\Delta \nu}{\nu}=(1+\alpha) \frac{\Delta U}{c^{2}} \quad\left(U=-\Phi_{\mathrm{eff}}\right),
$$

Hence we find that $\alpha \approx \delta^{2}$.

Numerous experiments were performed to check the formula (44) ( 17]). Without further discussion of the experimental data obtained, we notice that a reliable upper bound of $\alpha$ is given by [1]: $|\alpha|<2 \cdot 10^{-4}$. Hence we obtain a first estimate for $\bar{\sigma}$ at the present time, following from the red shift experiments:

$$
\delta^{2}<2 \cdot 10^{-4} \quad \text { or } \quad \frac{f^{\prime}(\bar{\sigma})}{f(\bar{\sigma})}=\delta<1.4 \cdot 10^{-2} .
$$

\subsection{The first post-Newtonian approximation}

Using the solution of the field equations in Newtonian-like approximation (see the next section) we claim that the expansion of the $s$ starts with a term of the order $\sim \beta^{2}$ :

$$
\sigma=\bar{\sigma}+\frac{1}{c^{2}} \Lambda+\frac{1}{c^{4}} \lambda+O\left(\frac{1}{c^{6}}\right) .
$$

Substituting the expansion series (29), (30) and (46) in the field equations and taking into account the explicit expression (5) for the stress-energy tensor, we receive a set of equations, which simply may be integrated in so-called harmonic coordinates defined by $\Gamma_{j m}^{i} g^{j m}=0$. For simplicity omitting the intermediate results, we finally arrive at the outcome:

$$
\begin{aligned}
& g_{\alpha \beta}=\delta_{\alpha \beta}\left(1-\frac{2 \phi}{c^{2}}\right)+O\left(\beta^{4}\right), \\
& g_{\alpha 4}=\frac{1}{c^{3}} \xi_{\alpha}+O\left(\beta^{5}\right), \\
& g_{44}=-\left[1+\frac{2 \phi}{c^{2}}+\frac{2}{c^{4}}\left(\phi^{2}+\chi\right)\right]+O\left(\beta^{6}\right),
\end{aligned}
$$


where the abbreviations

$$
\begin{gathered}
\phi(\boldsymbol{x}, t)=-\gamma_{\mathrm{N}} \int \frac{\mu\left(\boldsymbol{x}^{\prime}, t\right)}{\left|\boldsymbol{x}-\boldsymbol{x}^{\prime}\right|} d^{3} \boldsymbol{x}^{\prime}, \\
\xi_{\alpha}(\boldsymbol{x}, t)=-4 \gamma_{\mathrm{N}} \int \frac{\mu\left(\boldsymbol{x}^{\prime}, t\right) v_{\alpha}\left(\boldsymbol{x}^{\prime}, t\right)}{\left|\boldsymbol{x}-\boldsymbol{x}^{\prime}\right|} d^{3} \boldsymbol{x}^{\prime}, \\
\chi=\Phi_{3}-2 \Phi_{2}+2 \Phi_{1}+3 \Phi_{4}, \\
\Phi_{1}(\boldsymbol{x}, t)=-\gamma_{\mathrm{N}} \int \frac{\mu\left(\boldsymbol{x}^{\prime}, t\right) v^{2}\left(\boldsymbol{x}^{\prime}, t\right)}{\left|\boldsymbol{x}-\boldsymbol{x}^{\prime}\right|} d^{3} \boldsymbol{x}^{\prime}, \\
\Phi_{2}(\boldsymbol{x}, t)=-\gamma_{\mathrm{N}} \int \frac{\mu\left(\boldsymbol{x}^{\prime}, t\right) \phi\left(\boldsymbol{x}^{\prime}, t\right)}{\left|\boldsymbol{x}-\boldsymbol{x}^{\prime}\right|} d^{3} \boldsymbol{x}^{\prime}, \\
\Phi_{3}(\boldsymbol{x}, t)=-\frac{1}{4 \pi} \int \frac{\partial^{2} \phi\left(\boldsymbol{x}^{\prime}, t\right)}{\partial t^{2}} \frac{1}{\left|\boldsymbol{x}-\boldsymbol{x}^{\prime}\right|} d^{3} \boldsymbol{x}^{\prime}, \\
\vartheta=c_{4}(\boldsymbol{x}, t)=-\gamma_{\mathrm{N}} \int \frac{p\left(\boldsymbol{x}^{\prime}, t\right)}{\left|\boldsymbol{x}-\boldsymbol{x}^{\prime}\right|} d^{3} \boldsymbol{x}^{\prime}, \\
\left.\sigma 1+\frac{\phi}{c^{2}} \delta^{2}+\frac{1}{c^{4}}\left(\delta^{4} \phi^{2}-\lambda \delta\right)+O\left(1 / c^{6}\right)\right], \\
\bar{\sigma}\left\{1-\frac{\delta^{2}}{c^{2}} \phi-\frac{\delta^{2}}{c^{4}}\left[\chi-2 \Phi_{1}-3 \Phi_{4}\right]+O\left(1 / c^{6}\right)\right\},
\end{gathered}
$$

were used. Here $\mu$ and $p$ are mass density and pressure respectively.

In the preceding relations, as well as in the subsequent equations of motion

for a neutral test particle, the terms of the order of magnitude $\frac{v^{2}}{c^{2}} \delta^{4}$ were neglected:

$$
\begin{aligned}
\frac{\mathrm{d} \boldsymbol{v}}{\mathrm{d} t} & =-\left(1-\delta^{2}\right) \boldsymbol{\nabla}\left(\phi+\frac{2}{c^{2}} \phi^{2}+\frac{1}{c^{2}} \chi\right) \\
& -\left(1+\delta^{2}\right) \frac{v^{2}}{c^{2}} \boldsymbol{\nabla} \phi+3 \frac{\boldsymbol{v}}{c^{2}} \frac{\partial \phi}{\partial t}\left(1+3 \delta^{2}\right) \\
& -\frac{1}{c^{2}} \frac{\partial \boldsymbol{\xi}}{\partial t}+\frac{\boldsymbol{v}}{c^{2}} \times(\boldsymbol{\nabla} \times \boldsymbol{\xi})+\frac{4 \boldsymbol{v}}{c^{2}}(\boldsymbol{v} \boldsymbol{\nabla}) \phi \\
& -\frac{\delta^{2}}{c^{2}} \boldsymbol{\nabla}\left(2 \Phi_{1}+3 \Phi_{4}\right)+O\left(\beta^{4}\right) .
\end{aligned}
$$

\subsection{The equations of motion of $N$ gravitationally bounded point-like bodies in the first post-Newtonian approx- imation}

For the first time the approximated equations of motion within the framework of the Einstein theory were derived by A. Einstein, L. Infeld and B. Hoffmann 
(1938) as well later by a different approach by V. Fock and N. M. Petrova (1939, 1949). These publications initiated the start of a lot of papers which mainly aimed at the derivation of the equations of motion from the field equations.

As already mentioned above, the equations of motion can be obtained from the field equations of PUFT in the same way as in the Einstein theory. Omitting intermediate results, now for the $N$ point-like bodies (compare with (10) and (11)) we present the substrate stress tensor $\Theta^{m n}$ and the scalmass density $\mu^{(\sigma)}$ :

$$
\begin{aligned}
& \Theta^{m n}(\boldsymbol{r})=-\sum_{A=1}^{A=N} M_{A} \frac{1}{\sqrt{-g}} \frac{\mathrm{d} x_{A}^{m}}{\mathrm{~d} t} \frac{\mathrm{d} x_{A}^{n}}{\mathrm{~d} t} \frac{\mathrm{d} t}{\mathrm{~d} \tau} \delta^{(3)}\left(\boldsymbol{r}-\boldsymbol{r}_{A}\right), \\
& \mu^{(\sigma)} \equiv \frac{1}{c^{2}} \vartheta=\sum_{A=1}^{A=N} \mathcal{M}_{A} \frac{1}{\sqrt{-g}} \frac{\mathrm{d} t}{\mathrm{~d} \tau} \delta^{(3)}\left(\boldsymbol{r}-\boldsymbol{r}_{A}\right)
\end{aligned}
$$

Taking into account the explicit expression (47) for the metric, then we find in the first post-Newtonian approximation the following expressions for the mass density and the scalmass density:

$$
\begin{gathered}
\mu(\boldsymbol{r})=\sum_{A} M_{A}\left(1-\frac{v_{A}^{2}}{2 c^{2}}+\frac{3 \phi}{c^{2}}\right) \delta^{(3)}\left(\boldsymbol{r}-\boldsymbol{r}_{\boldsymbol{A}}\right)+O\left(\beta^{4}\right), \\
\mu^{(\sigma)}(\boldsymbol{r})=\sum_{A} \mathcal{M}_{A}\left(1-\frac{v_{A}^{2}}{2 c^{2}}+\frac{3 \phi}{c^{2}}\right) \delta^{(3)}\left(\boldsymbol{r}-\boldsymbol{r}_{\boldsymbol{A}}\right)+O\left(\beta^{4}\right) .
\end{gathered}
$$

Hence in this case the equations (8) lead to the following equations of motion for the gravitationally bounded $N$ point-like bodies within the framework of PUFT:

$$
\begin{aligned}
\frac{\mathrm{d} \boldsymbol{v}_{\boldsymbol{K}}}{\mathrm{d} t} & =\sum_{A \neq K} \frac{\gamma_{\mathrm{N}} M_{A} \boldsymbol{r}_{A K}}{r_{A K}^{3}}\left[( 1 - \delta ^ { 2 } ) \left(1-4 \sum_{B \neq K} \frac{\gamma_{\mathrm{N}} M_{B}}{r_{B K} c^{2}}\right.\right. \\
& \left.-\sum_{C \neq A} \frac{\gamma_{\mathrm{N}} M_{C}}{r_{C A} c^{2}}\left(1-\frac{\boldsymbol{r}_{A K} \cdot \boldsymbol{r}_{C A}}{2 r_{C A}^{2}}\right)-\frac{3}{2 c^{2}}\left(\frac{\boldsymbol{v}_{\boldsymbol{A}} \cdot \boldsymbol{r}_{\boldsymbol{A}}}{r_{A K}}\right)^{2}\right) \\
& \left.+\left(1+\delta^{2}\right) v_{K}^{2} / c^{2}+2 v_{A}^{2} / c^{2}-4 \boldsymbol{v}_{K} \cdot \boldsymbol{v}_{A} / c^{2}\right] \\
& +\frac{1}{2}\left(7+\delta^{2}\right) \sum_{A \neq K} \sum_{C \neq A} \boldsymbol{r}_{C A} \frac{\gamma_{\mathrm{N}}^{2} M_{A} M_{C}}{r_{A K} r_{C A}^{3} c^{2}} \\
& -\sum_{A \neq K}\left(\boldsymbol{v}_{A}-\boldsymbol{v}_{K}\right) \frac{\gamma_{\mathrm{N}} M_{A} \boldsymbol{r}_{A K} \cdot\left[\left(3+\delta^{2}\right) \boldsymbol{v}_{A}-4 \boldsymbol{v}_{K}\right]}{c^{2} r_{A K}^{3}}
\end{aligned}
$$

with $\gamma_{\mathrm{N}}=\left(1+\delta^{2}\right) G_{\mathrm{S}}=$ const.

This system of equations of motion can be considered as the analog (in PUFT) to the Einstein-Infeld-Hoffmann equations (in the Einstein theory). 


\subsection{Perihelion Motion of Mercury}

In this section we investigate the motion of a test body (e.g. Mercury) around a central body (e.g. sun) which for simplicity will be considered as non-rotating and spherically symmetric. As before we suggest that the condition $\delta \ll 1$ is fulfilled. Under these assumptions the integration of the field equations leads to

$$
\begin{aligned}
& \text { a) } g_{\alpha \beta}=\delta_{\alpha \beta}\left(1+\frac{2 \gamma_{\mathrm{N}} M_{c}}{c^{2} R}\right)+O\left(\beta^{4}\right) \text {, } \\
& \text { b) } g_{\alpha 4}=0 \text {, } \\
& \text { c) } g_{44}=-1+\frac{2 \gamma_{\mathrm{N}} M_{c}}{c^{2} R}-\frac{2 \gamma_{\mathrm{N}}^{2} M_{c}^{2}}{c^{4} R^{2}}+O\left(\beta^{6}\right) \text {, }
\end{aligned}
$$

where $M_{c}$ is a constant coinciding with the inertial mass of the central body if the scalaric field vanishes. Therefore in this approximation the metric has the same form as the metric in the Einstein theory. We have to note that E. Schmutzer 2, 18, succeeded in finding the exact spherically symmetric solutions in implicit form. In the paper [19] we find three parametric exact spherically symmetric solution in explicit form. In this paper we find this solution in the harmonic coordinates, which were used by obtaining the post-Newtonian equations. This solution in the corresponding approximation is identically to (63).

The equation of motion of the test body reads:

$$
\begin{aligned}
\frac{\mathrm{d}^{2} \boldsymbol{R}}{\mathrm{d} t^{2}} & \equiv \frac{\mathrm{d} \boldsymbol{v}}{\mathrm{d} t}=-\frac{\gamma_{\mathrm{N}} M_{c}}{R^{3}} \boldsymbol{R}\left[\left(1-\delta^{2}\right)\left(1-\frac{4 \gamma_{\mathrm{N}} M_{c}}{c^{2} R}\right)\right. \\
& \left.+\left(1+\delta^{2}\right) \frac{v^{2}}{c^{2}}\right]+\frac{4 \gamma_{\mathrm{N}} M_{c}}{c^{2} R^{3}} \boldsymbol{v}(\boldsymbol{v} \boldsymbol{R})+O\left(\beta^{4}\right) .
\end{aligned}
$$

In the Newtonian approximation the equation (64) goes over into the equation of motion

$$
\frac{\mathrm{d}^{2} \boldsymbol{R}}{\mathrm{d} t^{2}} \equiv \frac{\mathrm{d} \boldsymbol{v}}{\mathrm{d} t}=-\frac{\gamma_{\mathrm{N}} M_{c}}{R^{3}} \boldsymbol{R}\left(1-\delta^{2}\right) .
$$

Integration leads to the following set of equations:

$$
\begin{gathered}
R^{2} \frac{\mathrm{d} \varphi}{\mathrm{d} t}=\left[\gamma_{\mathrm{N}} M_{c}\left(1-\delta^{2}\right) P\right]^{1 / 2} \\
\boldsymbol{v} \equiv \frac{\mathrm{d} \boldsymbol{R}}{\mathrm{d} t}=\left[\frac{\gamma_{\mathrm{N}} M_{c}\left(1-\delta^{2}\right)}{P}\right]^{1 / 2}\left[-\boldsymbol{e}_{x} \sin \varphi+\boldsymbol{e}_{y}(\varepsilon+\cos \varphi)\right]
\end{gathered}
$$

where
a) $\quad \boldsymbol{R}=R\left(\boldsymbol{e}_{x} \cos \varphi+\boldsymbol{e}_{y} \sin \varphi\right)$
b) $R=P(1+\varepsilon \cos \varphi)^{-1}$.

As it is well-known, this solution describes the motion of the test body in a plane (spanned by the basis vectors $\boldsymbol{e}_{x}$ and $\boldsymbol{e}_{y}$ ) orthogonal to the angular momentum. 
We remind that $R$ and $\varphi$ are polar coordinates in the plane of motion, $P$ and $\varepsilon$ (eccentricity) are the parameters of the ellipse.

Applying the method of successive approximation we find the following postNewtonian solution:

$$
\begin{gathered}
\boldsymbol{v}=\left[\frac{\gamma_{\mathrm{N}} M_{c}\left(1-\delta^{2}\right)}{P}\right]^{1 / 2}\left[-\boldsymbol{e}_{x} \sin \varphi+\boldsymbol{e}_{y}(\varepsilon+\cos \varphi)\right]+\delta \boldsymbol{v}, \\
R^{2} \frac{\mathrm{d} \varphi}{\mathrm{d} t} \equiv(\boldsymbol{R} \times \boldsymbol{v})_{z}=\left[\gamma_{\mathrm{N}} M_{c}\left(1-\delta^{2}\right) P\right]^{1 / 2}(1+\delta h),
\end{gathered}
$$

where

$$
\delta h=-\frac{4 \gamma_{\mathrm{N}} M_{c}}{c^{2} P} \varepsilon \cos \varphi
$$

and

$$
\begin{aligned}
\delta \boldsymbol{v} & =\sqrt{\frac{\gamma_{\mathrm{N}} M_{c}\left(1-\delta^{2}\right)}{P}}\left(\frac{\gamma_{\mathrm{N}} M_{c}}{c^{2} P}\right)\left\{\boldsymbol { e } _ { x } \left[\sin \varphi\left(\left(3-\varepsilon^{2}\right)-\delta^{2}\left(1+\varepsilon^{2}\right)\right)\right.\right. \\
& \left.-\varepsilon\left(3+\delta^{2}\right) \varphi+\frac{1}{2} \varepsilon\left(1-\delta^{2}\right) \sin 2 \varphi\right] \\
& \left.-\boldsymbol{e}_{y}\left[\cos \varphi\left(1+\varepsilon^{2}\right)\left(3-\delta^{2}\right)+\frac{\varepsilon}{2}\left(1-\delta^{2}\right) \cos 2 \varphi\right]\right\} .
\end{aligned}
$$

Using the identity

$$
\frac{\mathrm{d}}{\mathrm{d} \varphi} \frac{1}{R}=-\left(R^{2} \frac{\mathrm{d} \varphi}{\mathrm{d} t}\right)^{-1}\left(\frac{\boldsymbol{R} \boldsymbol{v}}{R}\right)
$$

we get from (72) for the trajectory of the test body:

$$
\begin{aligned}
\frac{P}{R} & =1+\varepsilon \cos \varphi+\frac{\gamma_{\mathrm{N}} M_{c}}{c^{2} P}\left[\frac{1}{2} \varepsilon \cos \varphi\left(7+\delta^{2}\right)\right. \\
& \left.+\varepsilon\left(3+\delta^{2}\right) \varphi \sin \varphi\right] .
\end{aligned}
$$

The calculation of the perihelion motion leads to the result

$$
\delta \varphi \approx \frac{6 \pi \gamma_{\mathrm{N}} M_{c}}{c^{2} P}\left(1+\frac{1}{3} \delta^{2}\right)
$$

for one revolution. This formula (75) is identical with the relation found by Schmutzer 3 using the exact spherically symmetric solution. If $\delta=0$, then the relation (75) coincides with the corresponding result in the Einstein theory.

Finally to this section 3 we draw the attention of the reader at a new paper by E. Schmutzer on a set of possibilities to couple scalarism $(\vartheta)$ to the usual 4-dimensional physics, being investigated by him [20]. 


\section{Conclusion}

In the present paper we investigated the equation of motion of a point-like test body in PUFT and the possibility of functional dependence of the inertial mass of an external scalaric field. Although the idea of variability of inertial mass is not new itself ( see e.g. 21]), in PUFT it appears quite natural.

Let us remind that in PUFT the gravitational central mass $M_{c}$ appears in the exact spherically symmetric solution. At the same time the acceleration of test bodies in an external gravitational field is characterized by the effective Newtonian potential $\Phi_{\text {eff }}$ (see (41) and (42)). Using the definition (39) we obtain for the solar system that $M_{c}=M_{\odot}$, since the mass of the sun has to be determined by its gravitational interaction $\left(\Phi_{\mathrm{eff}}=-G_{\mathrm{S}} M_{\odot} / r\right)$. Hence follows that the perihelion motion of Mercury reads

$$
\delta \varphi \approx \frac{6 \pi G_{\mathrm{S}} M_{\odot}}{c^{2} P}\left(1+\frac{4}{3} \delta^{2}\right) .
$$

The Einstein effects, as light deflection and photon frequency shift, are determined exclusively by the space-time geometry. Therefore the results in PUFT coincide with the corresponding ones in the Einstein theory if $M_{c}$ is used. Expressing the mass $M_{c}$ by the experimentally determined value of the solar mass $M_{\odot}$, we obtain the following formulas for the light deflection:

$$
\triangle \chi=\frac{4 G_{\mathrm{S}}}{R_{\odot} c^{2}} M_{\odot}\left(1+\delta^{2}\right),
$$

where $R_{\odot}$ is the radius of the Sun. Of course (76) and (77) can be applied for calculation of post-Newtonian effects only if corrections connected with $\delta^{2}$ are greater than the post-post-Newtonian ones. If we compare the two last expressions with the experiment (see [1]) then we find immediately that from (76) and (77) follows that $\delta<0.048$ and $\delta<10 \cdot 10^{-2}$, respectively. Let's remind that from the red shift experiment follows (see (45)): $\delta<1.4 \cdot 10^{-2}$.

\section{Acknowledgement}

We are very grateful to professor M. Schneider (formerly Technical University of Munich) for his intended comparison of PUFT with measuring astrophysics, that has motivated us to this approximate treatment of PUFT.

A. K. Gorbatsievich is very grateful to DAAD and FSU Jena (Germany) for financial support and hospitality.

\section{References}

[1] C. M. Will, Living Rev. Relativity 9 (2006), 3 (www.livingreviews.org/lrr2006-3); ArXiv gr-qc/0510072).

[2] E. Schmutzer, Fortschr. Phys. 43, (1995) 613. 
[3] E. Schmutzer, Projektive Einheitliche Feldtheorie mit Anwendungen in Kosmologie und Astrophysik. Neues Weltbild ohne Urknall? (Mit einem Anhang von A. K. Gorbatsievich) (Verlag Harri Deutsch GmbH, Frankfurt am Main, 2004).

[4] E. Schmutzer, Fünfdimensionale Physik (Wissenschaftsverlag Thüringen, Langewiesen 2009).

[5] E. Schmutzer, Gen. Rel. Gravit. 33 (2001) 843.

[6] E. Schmutzer, J. Mod. Phys. E 16 (2007) 1181.

[7] E. Schmutzer, J. Mod. Phys. E 18 (2009) 1903.

[8] A. K. Gorbatsievich, Gen. Rel. Grav. 33, (2001) 965.

[9] E. Schmutzer, in: Proceedings of the 17th Erice Course of the International School of Cosmology and Gravitation, (Eds. P.G. Bergmann and V. de Sabbata, Kluwer Academic Publishers, Dordrecht, 2002), p. 387

[10] A. A. Blinkouski, and A. K. Gorbatsievich, Gravitation and Cosmology, 7 (2001) 286.

[11] E. Schmutzer, Habilatationsschrift at the Friedrich Schiller University of Jena, 1958.

[12] A. K. Gorbatsievich, Ho Si Mau Tchuc, and E. Schmutzer, Acta Phys. Pol., B 27 (1996) 1991.

[13] E. V. Pitjeva, Astronomy Letters 31 (2005b) 940.

[14] A. J. Sanders, G. T. Gillies, and E. Schmutzer, Ann. Phys. (Berlin), 522 (2010) 861.

[15] R.V. Pound, and G.A. Rebka, Phys. Rev. Lett. 4 (1960) 337.

[16] R.V. Pound, and J.L. Snider, Phys. Rev., B140 (1965) 788.

[17] Will, C. M., Theory and experiment in gravitational physics (Cambridge University Press, Cambridge, U.K.; New York, U.S.A., 1993, 2nd edition).

[18] E. Schmutzer, Ann. Physik. 4 (1995) 251.

[19] A. K. Gorbatsievich, in: The Gravitation Constant: Generalized Gravitational Theories and Experiments, NATO Science Series (eds. De Sabbata, T. Gillies, and V. Melnikov, Kluwer Academic Publishers, Dordrecht, Boston, London, 2004) p. 192.

[20] E. Schmutzer, in: Schriften der Sudetendeutschen Akademie der Wissenschaften und Künste, 31 (München, 2011) p. 123.

[21] J.D. Bekenstein, Phys. Rev. D15, (1977) 1458. 